\title{
Avaliação da uniformidade de peso e de doses de ranitidina em cápsulas magistrais
}

\author{
Mean weight and content uniformity of ranitidine capsules
}

Recebido em: 06/02/2017

Aceito em: $\quad 14 / 05 / 2017$

\author{
Bruna do Carmo BARQUETTE; Mariana Coleta Campolina BELO; \\ Rafaela Mafia da Cruz SILVA; André Lima de Oliveira COSTA \\ Curso de Farmácia. Centro Universitário UNA. Rua dos Guajajaras, 175 - Centro, CEP \\ 30180-100.Belo Horizonte, MG, Brasil.E-mail: sodioepotassio@hotmail.com
}

\section{ABSTRACT}

Brazilian compounding pharmacies should follow good manufacturing practices defined by Anvisa to ensure the efficacy, safety and credibility of medicines dispensed to the population. The pharmacopoeial analyzes have limited application in compounding pharmacies. However, minimal tests, as weight determination of capsules, are required for the quality control of medicines preparations. Other tests are applicable in cases of minimum stock or periodical analysis, to monitoring the manufacturing process. In this study, of mean weight and uniformity of dosage forms were evaluated for ranitidine capsules from six different compounding pharmacies in Belo Horizonte, MG, Brasil. The dosage forms were analyzed according to Brazilian Pharmacopoeia and National Formulary. Only one pharmacy presented capsules with an unsatisfactory result in the mean weight test. In uniformity of dosage forms, all analysed capsules were disapproved in the test criteria. The results showed that capsules with uniformity of weight do not necessarily present a uniformity distribution of the drug. The control of manufacturing process is essential for ensuring quality, effective and safe products.

Keywords: quality; compounding pharmacy; ranitidine; uniformity of dosage forms; mean weight.

\section{RESUMO}

Para garantir a eficácia, a segurança e a credibilidade aos medicamentos dispensados à população, as farmácias magistrais devem aplicar as Boas Práticas de Manipulação, de acordo com a Anvisa. Ainda que limitada a aplicação integral das análises farmacopeicas nas farmácias de manipulação, a resolução estabelece os ensaios mínimos exigidos ao controle da qualidade das preparações magistrais e oficinais. Demais testes são aplicáveis nos casos de manipulação de estoque mínimo ou análises trimestrais como forma de monitoramento do processo magistral. Neste estudo, a uniformidade de peso e de conteúdo foram avaliadas em cápsulas de ranitidina de seis diferentes farmácias magistrais em Belo Horizonte, MG, Brasil. As preparações foram analisadas de acordo com os procedimentos de determinação de peso e de uniformidade de doses unitárias preconizados na Farmacopeia Brasileira e no Formulário Nacional. Apenas uma farmácia apresentou cápsulas com resultado insatisfatório no teste de determinação de peso. Em relação à uniformidade de doses, os produtos de todas as farmácias foram reprovados nos critérios do teste. Os resultados mostram que as cápsulas com uniformidade de peso não apresentam, necessariamente, uma distribuição homogênea do princípio ativo. O controle rigoroso do processo de manipulação é essencial para a garantia de produtos de qualidade, eficazes e seguros.

Palavras-chave: qualidade, manipulação, ranitidina, uniformidade de doses, peso médio. 


\section{INTRODUÇÃO}

A ranitidina foi resultado de um desenvolvimento racional de fármacos, com base na relação estrutura-atividade, com vista à inibição competitiva da histamina nos receptores $\mathrm{H}_{2}$ das células parietais. Ela é tradicionalmente indicada no tratamento de úlcera gástrica e duodenal, esofagite erosiva e refluxo gastroesofágico, pois reduz a secreção ácida gástrica durante o dia e em condições basais noturnas, mesmo quando estimulada por alimentos, insulina, aminoácidos, histamina ou pentagastrina. O fármaco tem ação de longa duração (12 horas), sendo usualmente administrado em dose única diária de $150 \mathrm{mg}$ de ranitidina, na forma do sal cloridrato (1-5).

Atualmente, muitos pacientes recorrem às farmácias de manipulação para aquisição dos medicamentos. Os estudos envolvendo medicamentos manipulados demonstram elevados índices de satisfação dos usuários desses medicamentos $(6,7)$. O nível de satisfação desses usuários pode ser explicado pelas vantagens que esses produtos oferecem, como o tratamento individualizado, a associação de vários princípios ativos em uma única formulação e a adequação de doses terapêuticas. Por outro lado, a falta de confiança nos medicamentos magistrais pode ocorrer pela dúvida da população quanto à eficácia e à segurança desses produtos, devido à falta de esclarecimentos acerca do processo produtivo dos medicamentos manipulados $(7,8)$.

A qualidade do medicamento, o atendimento e o tempo de dispensação são atributos valorizados pelos clientes de uma farmácia de manipulação. Normalmente os clientes esperam que o atendimento seja qualificado, orientativo e esclarecedor de questionamentos (9). Nesse contexto, cabe ao farmacêutico exercer essas funções. Ele atua como responsável técnico da farmácia de manipulação e a sua presença no estabelecimento é fundamental na produção de medicamentos de qualidade, na orientação e, consequentemente, na fidelização dos clientes.

No intuito de garantir a qualidade dos produtos manipulados, a Resolução da Diretoria Colegiada (RDC) nº7/2007 da Agência Nacional de Vigilância Sanitária (Anvisa), estabelece os ensaios mínimos obrigatórios às farmácias magistrais, a exemplo do peso médio em preparações sólidas. Outros ensaios de qualidade, como teor e uniformidade de dose unitárias, são exigidos somente nos casos de manipulação de estoque mínimo e avaliações trimestrais do processo magistral (10).

Neste estudo foram avaliadas as cápsulas de ranitidina $150 \mathrm{mg}$ manipuladas por seis diferentes farmácias magistrais em Belo Horizonte, Minas Gerais, Brasil. O objetivo foi verificar o desempenho do processo magistral no que diz respeito à uniformidade do peso e de doses unitárias dos produtos.

\section{MATERIAL E MÉTODOS:}

Foram analisadas 180 cápsulas de ranitidina 150 $\mathrm{mg}$, obtidas de 6 diferentes farmácias de manipulação (F1 a F6), localizadas em Belo Horizonte, MG, no período de setembro e outubro de 2016. A uniformidade de peso e de dose das preparações foi avaliada de acordo com os procedimentos de determinação de peso e de uniformidade de doses unitárias, preconizados na Farmacopeia Brasileira (11) e no Formulário Nacional (12).

Determinação de peso - Formulário Nacional. O ensaio foi realizado a partir da análise do peso médio das cápsulas e o cálculo do respectivo desvio padrão relativo das medições. O peso médio é a média da pesagem individual de 10 unidades de cápsulas manipuladas. $\mathrm{O}$ desvio padrão relativo (DPR) não deve ser maior que $4 \%$ (12).

Determinação de peso - Farmacopeia Brasileira. Para a determinação de peso, foram pesadas 20 unidades individualmente e, após a remoção do conteúdo de cada cápsula, os invólucros vazios foram pesados. A partir da diferença entre os valores de massa das cápsulas cheias e vazias, dfoi determinado o peso médio do conteúdo e o desvio individual do conteúdo de cada cápsula em relação à média. São toleradas não mais que duas unidades fora do limite de $\pm 10 \%$, em relação ao peso médio do conteúdo, porém, nenhuma cápsula poderá estar acima ou abaixo do dobro da porcentagem indicada (11).

Uniformidade de doses unitárias. A uniformidade de doses unitárias pode ser realizada utilizando o método de variação de peso ou de uniformidade de conteúdo. A escolha do método depende da forma farmacêutica, da dose e da proporção do fármaco na forma farmacêutica unitária. O método de variação de peso proporciona uma estimativa da quantidade de fármaco em cada unidade, considerando o peso individual da unidade e o teor determinado ao lote. O método de uniformidade de conteúdo baseia-se na dosagem individual do fármaco em cada unidade posológica e pode ser aplicado em qualquer caso.

A uniformidade de doses unitárias das cápsulas de ranitidina foi avaliada pelo método de uniformidade de conteúdo. O teor individual de cada unidade foi determinado e o Valor de Aceitação (VA) calculado para cada 
produto, utilizando o valor de referência tabelado (M), a média do teor individual das unidades (X), a constante de aceitabilidade (k) e o desvio padrão (s), conforme a equação: $\mathrm{VA}=|\mathrm{M}-\mathrm{X}|+\mathrm{ks}$.

O produto cumpre o teste de uniformidade de doses unitárias se o VA calculado para as 10 primeiras unidades testadas não é maior que 15,0 . Se o VA for maior que 15,0 , são testadas mais 20 unidades. O produto cumpre o teste se o VA final calculado para as 30 unidades testadas não é maior que 15,0 e a quantidade de componente ativo de nenhuma unidade individual é menor que $(1-25$ $\times 0,01) \mathrm{M}$ ou maior que $(1+25 \times 0,01) \mathrm{M}(11)$.

Diversas técnicas analíticas são descritas para o doseamento de ranitidina (12-16). O teor de ranitidina no conteúdo das cápsulas foi realizado por espectrofotometria de absorção a $314 \mathrm{~nm}(11,17)$, a partir da construção de uma curva analítica. Para a construção da curva analítica, foi preparada uma solução estoque do padrão de trabalho de cloridrato de ranitidina na concentração equivalente a $125 \mu \mathrm{g} / \mathrm{mL}$ de ranitidina em água purificada. Em seguida, as diluições foram realizadas de forma a obter cinco níveis, em triplicata, no intervalo de 7,5 a $17,5 \mu \mathrm{g} / \mathrm{mL}$ de ranitidina (Tabela 1 ).

A solução amostra foi preparada transferindo o conteúdo da cápsula para balão volumétrico de 250 $\mathrm{mL}$ com auxílio de $30 \mathrm{~mL}$ de água purificada. Após agitação mecânica por 30 minutos, o volume do balão foi completado com o mesmo solvente. Foi feita diluição de $5 \mathrm{~mL}$ do filtrado da solução anterior para $250 \mathrm{~mL}$ com água purificada, de forma a obter uma solução aquosa na concentração teórica de $12,5 \mu \mathrm{g} /$ $\mathrm{mL}$ de ranitidina.

As análises foram realizadas no Laboratório do Centro Universitário UNA, equipado com balança Shimadzu AY220 e espectrofotômetro Femto 800 XI. Foi utilizado, como padrão de trabalho, cloridrato de ranitidina do fabricante Pharma Nostra, lote R-128PD0913, validade $08 / 2018$.

Tabela 1. Preparo das soluções diluídas de ranitidina em diferentes níveis de concentração $(7,5$ a $17,5 \mu \mathrm{g} / \mathrm{mL})$ para construção da curva analítica.

\begin{tabular}{|c|c|c|c|}
\hline Solução & Volume da solução estoque $(\mathrm{mL})$ & qsp $(\mathrm{mL})$ & Concentração final $(\mu \mathrm{g} / \mathrm{mL})$ \\
\hline 1 & 1,5 & 25,0 & 7,5 \\
\hline 2 & 2,0 & 25,0 & 10,0 \\
\hline 3 & 2,5 & 25,0 & 12,5 \\
\hline 4 & 3,0 & 25,0 & 15,0 \\
\hline 5 & 3,5 & 25,0 & 17,5 \\
\hline
\end{tabular}

\section{RESULTADOS E DISCUSSÃO}

As farmácias magistrais oferecem grande diversificação de produtos em função do atendimento das necessidades específicas de seus clientes. $\mathrm{O}$ grande desafio é a obtenção de medicamentos e produtos de qualidade, com garantia de eficácia terapêutica e de segurança ao paciente (18). As cápsulas representam a forma farmacêutica sólida mais produzida pelas farmácias de magistrais e possuem como desvantagem a dificuldade em conseguir uniformidade de peso, necessária para atender aos requisitos da garantia da qualidade (19).

O teste de determinação de peso permite verificar se as unidades de um mesmo lote apresentam uniformidade no peso. O peso médio é um indicador essencial para o controle da qualidade de rotina das farmácias magistrais, podendo demonstrar a ineficiência na técnica de manipulação empregada. A não conformidade nesse pa- râmetro constitui critério de reprovação do produto, excluindo a necessidade de execução de demais testes (2).

Garantir a dose unitária dos fármacos é relevante na qualidade dos produtos farmacêuticos, uma vez que a dose incorreta está diretamente relacionada ao aumento dos efeitos adversos, da toxicidade e de ineficácia terapêutica. $\mathrm{O}$ teste de uniformidade de doses unitárias permite avaliar a quantidade de princípio ativo presente nas unidades da forma farmacêutica a serem administradas no organismo (20).

Determinação de peso - Formulário Nacional. O Formulário Nacional (FN) é o código oficial brasileiro onde estão inscritas formulações farmacêuticas oficiais de uso consagrado, no intuito de padronizar e assegurar a qualidade da manipulação das mesmas (21).

O procedimento de determinação de peso é preconizado no FN a partir do cálculo do peso médio de 10 unidades íntegras e da análise do desvio padrão relativo 
das medições (12). Os produtos de todas as farmácias cumpriram com o requisito do teste, com exceção da farmácia F5, cujas cápsulas apresentaram valor de DPR acima de 4\% (Tabela 2). O valor elevado de DPR, nesse caso, é indicativo da falta de homogeneidade no peso das cápsulas, o que pode ocasionar diferenças de doses entre as unidades posológicas.

Tabela 2. Peso médio e desvio padrão relativo (DPR) de 10 unidades de cápsulas manipuladas por seis farmácias (F1 a F6) em Belo Horizonte, MG, Brasil

\begin{tabular}{|c|c|c|}
\hline Farmácia & Peso médio $(\mathbf{m g})$ & DPR $(\%)$ \\
\hline F1 & 267,12 & $1,95 \%$ \\
\hline F2 & 260,88 & $0,94 \%$ \\
\hline F3 & 237,54 & $1,69 \%$ \\
\hline F4 & 274,02 & $1,91 \%$ \\
\hline F5 & 259,23 & $5,20 \%$ \\
\hline F6 & 262,13 & $2,76 \%$ \\
\hline
\end{tabular}

As imprecisões na manipulação de cápsulas ocorrem pois as prescrições são feitas por unidade de massa e o processo de enchimento é realizado em função de volume. Como o peso varia em função da densidade, que difere a cada lote da matéria-prima produzida, diversas não-conformidades podem ocorrer se o cálculo do volume não for realizado corretamente (22).

No que diz respeito à operação de mistura de pós, é recomendado utilizar pós de tenuidades semelhantes e, se for preciso, proceder à trituração e à tamisação para homogeneizar o tamanho de partícula. A mistura deve ser iniciada com o pó de menor quantidade e sendo adicionados os demais conforme a ordem de quantidade crescente (23).

$\mathrm{O}$ uso de indicador de mistura (corante permitido) é útil quando há necessidade de misturar pequena quantidade de substância ativa a uma grande massa de excipiente. Nesse caso, também é importante adotar o princípio da diluição geométrica e, após a mistura dos pós, proceder a tamisação (24).

Em estudos citados por Defáveri (2012), os valores de DPR obtidos com os métodos de homogeneização das cápsulas utilizando gral e pistilo ou saco plástico, apresentaram valores abaixo de $2,5 \%$. Os melhores resultados foram obtidos com a homogeneização utilizando gral e pistilo (25).

Pinheiro (2008) analisou a variação de peso em diversas cápsulas. $\mathrm{O}$ pesquisador observou que a maioria das unidades apresentou valores de DPR inferior ou igual a $2,5 \%$ e, ainda, que quanto mais treinado era o manipulador, mais baixos eram os valores de DPR (26). Foi verificado, ainda, que nas preparações de cápsulas contendo sinvastatina, houve predominância de valores elevados de DPR, fato que pode ser justificado pela dificuldade de escoamento que a mistura de pós desse fármaco apresenta, o que dificulta seu encapsulamento.

Outro fator que contribui com a variação de peso de cápsulas manipuladas é a variação inerente dos invólucros das cápsulas duras. Essa variação pode ser associada à falta de qualificação dos fornecedores de matérias- primas para manipulação, considerando que esses devem attender a padrões de boas práticas de fabricação e serem fontes confiáveis no fornecimento dos insumos para as farmácias de manipulação.

O processo de encapsulamento dos pós também interfere na variação do peso dos produtos. $\mathrm{O}$ método mais utilizado para o preparo de cápsulas manipuladas é o de produção por nivelamento de superfície, sendo a seriedade da técnica e do operador fatores determinantes na distribuição dos pós de forma homogênea (27).

Para o enchimento de cápsulas existe uma grande variedade de modelos de encapsuladoras manuais, semi-automáticas e automáticas. As encapsuladoras manuais de policloreto de polivinila (PVC), encontradas majoritariamente nas farmácias magistrais, podem ser danificadas com o tempo de uso, comprometendo o produto acabado quanto à uniformidade de peso entre as unidades de cápsulas e diferenças de uniformidades nas doses (24).

Determinação de peso - Farmacopeia Brasileira. A Farmacopeia Brasileira é o código oficial farmacêutico do país, onde são estabelecidos os requisitos mínimos de qualidade para fármacos, insumos, drogas vegetais, medicamentos e produtos para a saúde. Tem por finalidade promover a saúde da população, a partir da definição dos requisitos de qualidade e segurança dos insumos para a saúde, além de apoiar as ações de regulação sanitária e induzir ao desenvolvimento científico e tecnológico nacional (11).

O procedimento de determinação de peso é estabelecido na Farmacopeia Brasileira $5^{\text {a }}$ Ed a partir do cálculo do peso do conteúdo de 20 unidades e da análise do desvio individual em relação ao peso médio do conteúdo (11). Os produtos de todas as farmácias avaliadas cumpriram com o requisito do teste, pois os conteúdos das cápsulas apresentaram valores dentro do intervalo de \pm $10 \%$ em relação ao peso médio do conteúdo (Figura 1). Mesmo o produto da farmácia F5, que apresentou uma 
unidade abaixo do desvio de $10 \%$, foi considerado satisfatório, poiso teste tolera no máximo duas unidades fora do limite especificado, porém nenhuma delas acima ou abaixo do dobro desse limite.

A partir dos diagramas de dispersão da Figura 1, é possível perceber a variabilidade dos processos magistrais das farmácias F1 a F6. A farmácia F2 apresentou a menor variação entre os pesos dos conteúdos das cápsulas, demonstrando um melhor desempenho na uniformidade entre unidades do lote. Ao contrário, a farmácia F5 teve elevada variação das medidas individuais em relação à média, o que está associado a deficiências no controle do processo magistral.

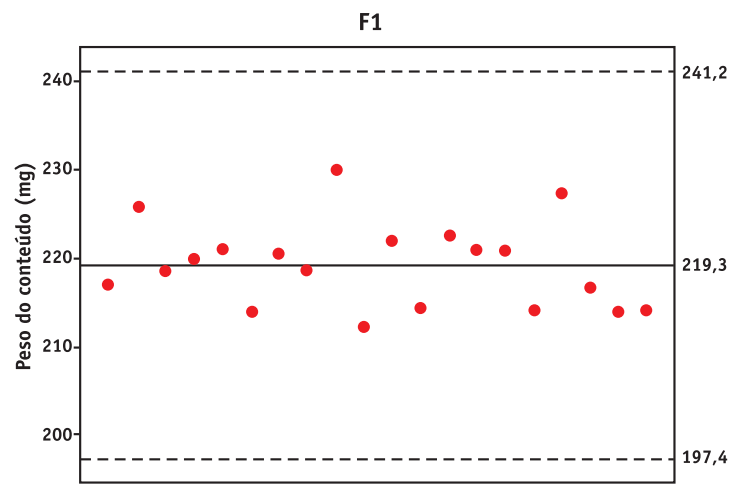

F3
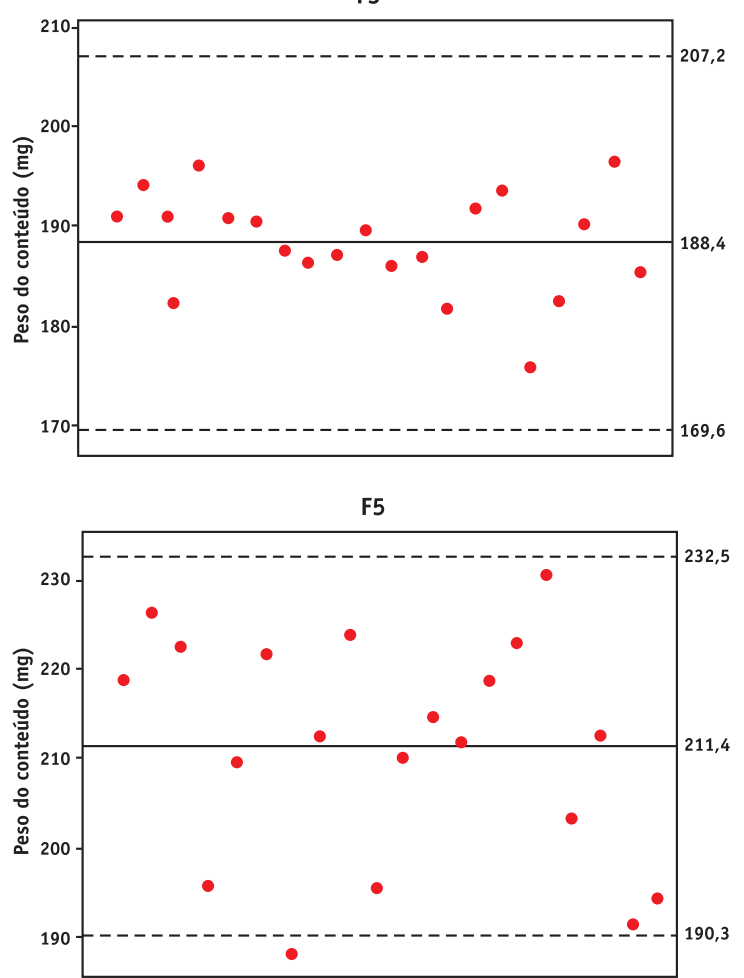

O teste de determinação de peso visa informar a homogeneidade do peso por unidades do lote, além de constituir-se de uma ferramenta importante no controle da qualidade de rotina das farmácias de manipulação (25). A aplicação da metodologia descrita na Farmacopeia Brasileira elimina possíveis desvios associados à variação do peso dos invólucros das cápsulas. Entretanto, ela não é viável no que se refere aos produtos magistrais, uma vez que requer a amostragem de 20 unidades e o teste é destrutível. A metodologia preconizada no Formulário Nacional garante a aplicabilidade do teste rotineiramente no âmbito das farmácias de manipulação, atendendo as exigências contidas na RDC 67/2007.
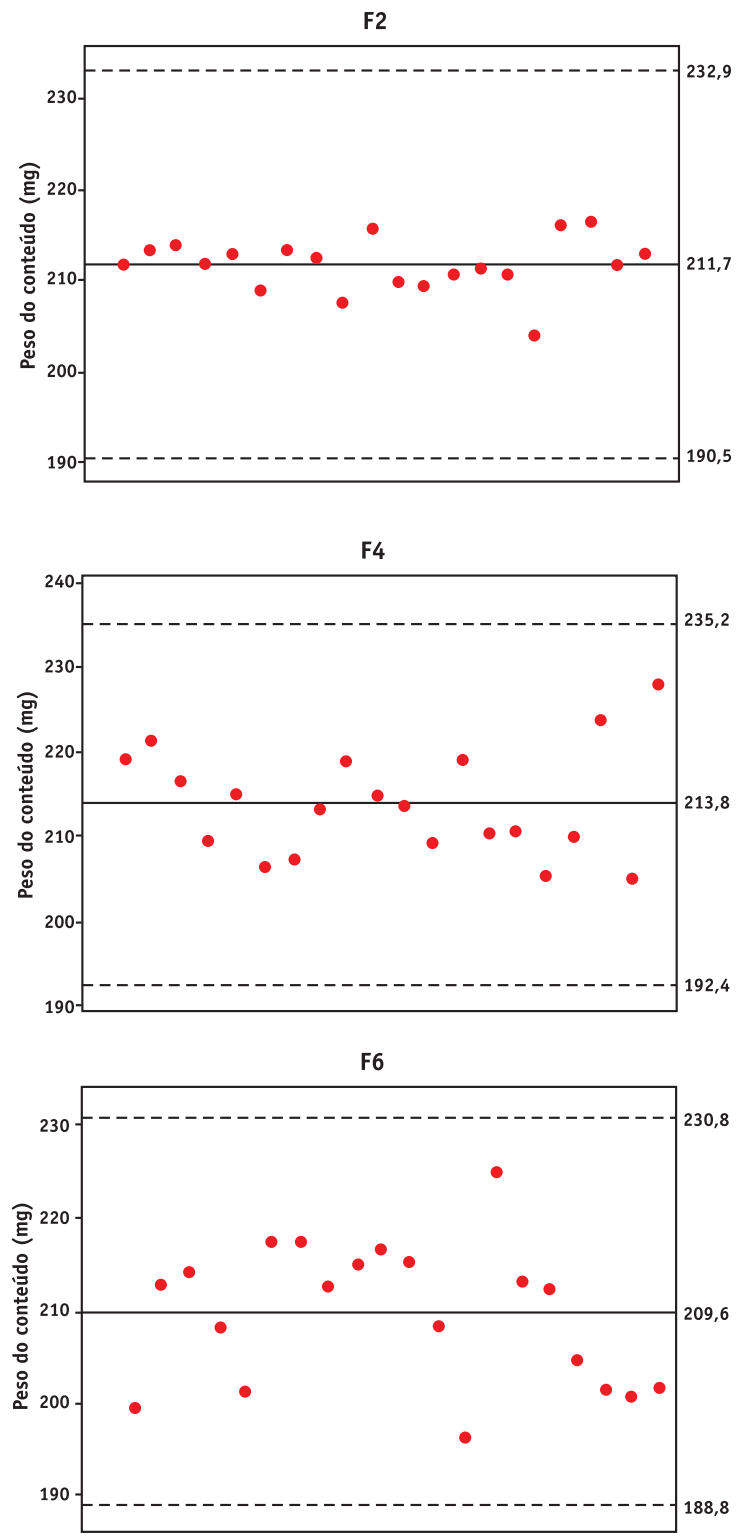

Figura 1. Gráficos de dispersão do peso do conteúdo de vinte cápsulas de ranitidina manipuladas por seis farmácias (F1 a F6), em Belo Horizonte, MG, Brasil. 
O cloridrato de ranitidina apresenta-se sob a forma de um pó cristalino, branco a amarelo pálido, inodoro, sensível à umidade. O fármaco tem faixa de fusão de $133{ }^{\circ} \mathrm{C}$ a $134^{\circ} \mathrm{C}$, é facilmente solúvel em água e em ácido acético (11). É importante ressaltar que não foram encontrados relatos na literatura de problemas farmacotécnicos no processo de encapsulamento do fármaco cloridrato de ranitidina.

Uniformidade de doses unitárias. $O$ teste de uniformidade de doses unitárias, preconizado nas farmacopeias, permite a avaliação da distribuição da quantidade de componente ativo entre as unidades de um lote. O produto cumpre o teste se o VA calculado não for maior que 15,0 .

Para a avaliação da uniformidade de conteúdo de ranitidina nas cápsulas manipuladas foi realizado o doseamento do fármaco em cada unidade, utilizando a técnica de espectrofotometria de absorção a $314 \mathrm{~nm}$ e a curva analítica construída no intervalo de 7,5 a 17,5 $\mu \mathrm{g} /$ $\mathrm{mL}$ (Figura 2). O método analítico empregado demonstrou linearidade satisfatória, de acordo com o parâmetro da Anvisa, que estabelece o critério mínimo aceitável de 0,99 para o coeficiente de correlação (r) (28). Por meio dos resultados obtidos, a seguinte equação da reta foi obtida: $\mathrm{y}=0,0451 \mathrm{x}-0,0521$ e ao valor do $\mathrm{r}^{2}=0,994$, sendo $\mathrm{r}=0,9970$.

Os produtos de todas as farmácias apresentaram valor de VA maior que 15,0 para as 10 primeiras unidades, sendo necessário testar mais 20 unidades. $O$ cálculo do VA para as 30 unidades também foi superior ao limite especificado para os produtos de todas as farmácias analisadas (Tabela 3). Além disso, as farmácias F5 e F6, apresentaram 3 e 2 unidades, respectivamente, com o teor abaixo do limite aceitável (73,88\%).

O cálculo do valor de aceitação (VA) leva em consideração dois parâmetros fundamentais: a média do teor do princípio ativo e o desvio padrão dos resultados obtidos. Para que o produto cumpra o teste é necessário que o teor médio das unidades esteja o mais próximo possível do valor rotulado $(100 \%)$ e as os teores individuais das unidades apresentem variabilidade controlada.

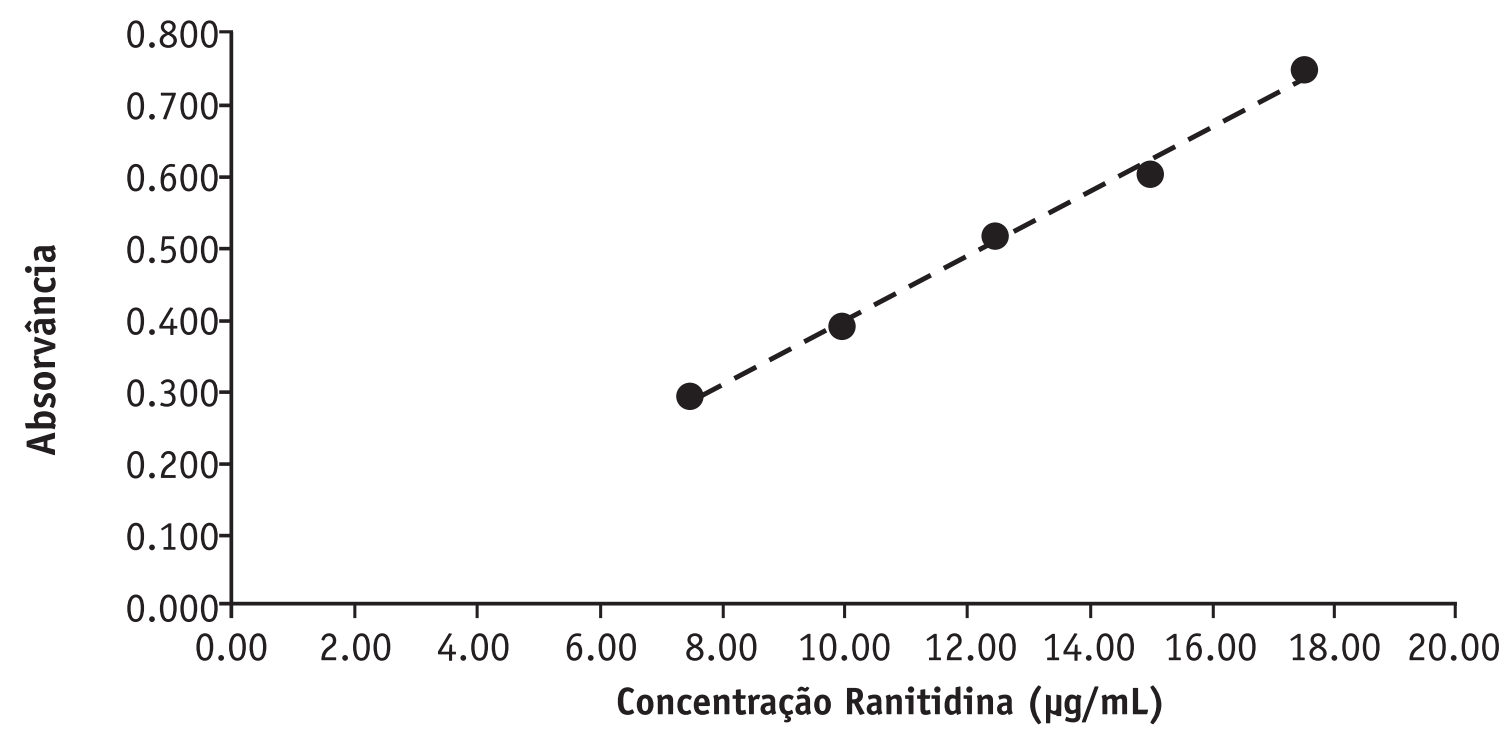

Figura 2. Curva analítica de ranitidina obtida na faixa de 7,5 a $17,5 \mu \mathrm{g} / \mathrm{mL}$ em meio aquoso por espectofotometria de absorção a $314 \mathrm{~nm}$.

Mesmo que as cápsulas tenham um peso uniforme, isso não garante que elas tenham a mesma dose, pois o processo de mistura pode não ser homogêneo. Na manipulação de cápsulas, a obtenção de uma mistura de pós bem definida e homogênea, a fim de garantir a dose adequada por unidade posológica é considerada uma etapa crítica nas farmácias magistrais, (29). A falta de uniformidade na distribuição dos fármacos pode resultar em unidades com sub-dose ou doses excessivas, contribuindo para ineficácia terapêutica ou efeitos adversos, respectivamente.

Diversos autores identificaram produtos manipulados com desvios de qualidade. Marcatto e cols (2005), encontraram cápsulas de captopril que não atendiam aos requisitos do teste de determinação de peso (30). Miotto Junior e Adams (2004), relataram 
a reprovação de cápsulas de nifedipino nos testes de uniformidade de conteúdo (31). Na avaliação da qualidade de cápsulas contendo $20 \mathrm{mg}$ de cloridrato de fluoxetina, Pissato e cols. (2006), mostraram que todos os produtos analisados foram aprovados no teste de determinação de peso, mas tiveram resultados insatisfatórios quanto à uniformidade de conteúdo (32).

Tabela 3. Média do teor de ranitidina expressa em porcentagem do valor rotulado (VR), desvio padrão (s) e cálculo do valor de aceitação (VA) de 10 e de 30 unidades de cápsulas manipuladas por seis farmácias (F1 a F6) em Belo Horizonte, MG, Brasil.

\begin{tabular}{|c|c|c|c|c|c|c|c|}
\multirow{2}{*}{ Farmácia } & \multicolumn{3}{|c|}{10 Unidades } & \multicolumn{3}{|c|}{30 Unidades } \\
\cline { 2 - 8 } & Média (\%VR) & S & VA & Média (\%VR) & S & 25,59 \\
\hline F1 & 113,19 & 5,22 & 31,74 & 113,68 & 4,84 & 19,42 \\
\hline F2 & 87,8 & 2,46 & 20,28 & 86,3 & 2,91 & 21,78 \\
\hline F3 & 89,67 & 4,01 & 26,8 & 92,48 & 6,17 & 16,68 \\
\hline F5 & 103,52 & 5,24 & 22,02 & 105,65 & 12,5 & 29,35 \\
\hline F6 & 92,41 & 13,49 & 28,03 & 91,18 & 7,51 & 27,65 \\
\hline
\end{tabular}

Na mesma linha de pesquisa, Meneghini e Adams (2007), realizaram um estudo sobre a qualidade de cápsulas contendo $5 \mathrm{mg}$ de diazepam (33). Os autores identificaram cápsulas com teor elevado e falta de uniformidade nas doses. Eles atribuíram os resultados insatisfatórios a possíveis erros de pesagem no início do processo de preparação. Consideraram também a possível ocorrência de segregação entre o pó dos excipientes e o pó da substância ativa durante o processo de mistura.

Sabe-se da importância da utilização do fator de equivalência sal e base para uma correta manipulação, já que uma substância ativa nem sempre pode ser utilizada na sua forma livre. Muitas vezes a formação de sais ou ésteres torna a substância mais efetiva e segura, com condições farmacocinéticas mais adequadas às necessidades dos pacientes (23).

Embora as prescrições sejam normalmente expressas em ranitidina, o fármaco terapeuticamente ativo é o cloridrato de ranitidina. Nesse caso, deve ser aplicado um fator de equivalência para calcular e expressar as doses no teor da base (ranitidina). Em uma prescrição de cápsulas de $150 \mathrm{mg}$ de ranitidina deve ser usado o fator de 1,12 para multiplicar a quantidade prescrita, a fim de ser obtida a quantidade de cloridrato de ranitidina em cada cápsula $(168 \mathrm{mg}$ ). Esse exemplo mostra a necessidade cada vez maior de padronização na forma de prescrever e expressar as doses, bem como as formas a serem utilizadas no preparo dos medicamentos (24).

\section{CONCLUSÃO}

As cápsulas de ranitidina de todas as farmácias avaliadas apresentaram resultados satisfatórios no teste de determinação de peso, conforme a metodologia preconizada na Farmacopeia Brasileira. Entretanto, os produtos não cumpriram com os requisitos do teste de uniformidade de doses unitárias.

Nas prescrições de ranitidina é importante considerar que o fármaco terapeuticamente ativo é o cloridrato de ranitidina. $\mathrm{O}$ uso do fator de equivalência deve ser aplicado nos cálculos farmacêuticos para garantir a manipulação correta das doses do medicamento.

Os procedimentos de mistura de pós e de encapsulação são etapas críticas na obtenção de cápsulas com uniformidade de peso e de doses. Assim, as farmácias magistrais devem controlar rigorosamente os processos de manipulação para garantir a qualidade do produto final. 


\section{REFERÊNCIAS}

1. BRASIL. Ministério da Saúde. Fundação Oswaldo Cruz. Brasil: Memento Terapêutico. Brasília 304p, 2006.

2. Santos ACCS. Desenvolvimento de Formulação de Ranitidina Comprimido e Comparação com Medicamento de Referência. [Dissertação]. Brasília: Universidade Católica de Brasília. 2011.

3. Coelho PMBS. Desenvolvimento de formulações de liberação modificada de ranitidina. [Dissertação]. Faculdade de Farmácia da Universidade do Porto. 2007.

4. Veevers AE, Oxberry SG. Ranitidine: forgotten drug of delayed gastric emptying. BMJ Support Palliat Care. 2016; DOI: 10.1136/bmjspcare-2016-001188.

5. Oztürk E, Yeşilova Z, Ilgan S, Ozgüven M, Dağalp K. Performance of acidified 14C-urea capsule breath test during pantoprazole and ranitidine treatment. J Gastroenterol Hepatol. 2009; 24(7):1248-1251. DOI: 10.1111/ j.1440-1746.2009.05845.

6. Alves AP, Moura AD, Neutgem ERV, Silva JM, Cunha SN, Oka SK, Machado SRP. Avaliação das boas práticas de manipulação nas farmácias com manipulação de Cuiabá e Várzea Grande, Estado de Mato Grosso. Rev. Bras. Farm. 2009; 90(1): 75-80.

7. Fernandes CKC. Análise do perfil de utilização de medicamentos manipulados no município de Goiânia/ GO. [Dissertação]. Goiânia: Mestrado em Desenvolvimento Regional - Faculdade Alves Faria. 2015.

8. Gindri AL, Souza LBD, Gindri LL, Gindri AL. Análise dos consumidores de drogarias e farmácias e índice de aceitação dos medicamentos manipulados na cidade de São Francisco de Assis, RS. Rev. Bras. Farm. 2013; 94 (2): 184-188.

9. Faria GGS. Atributos valorizados pelos consumidores de farmácia de manipulação residentes no bairro Glória. [Tese]. Porto Alegre: Departamento de Ciências Administrativas da Universidade Federal do Rio Grande do Sul. 2011.

10. BRASIL. Agência Nacional de Vigilância Sanitária. Resolução RDC $n^{\circ} 67$, de 8 de outubro de 2007. Dispõe sobre Boas Práticas de Manipulação de Preparações Magistrais e Oficinais para Uso Humano em farmácias. Diário Oficial da República Federativa do Brasil, 9 de outubro de 2007.

11. BRASIL. Farmacopeia Brasileira. $5^{\circ}$ edição. Agência Nacional de Vigilância Sanitária. Brasília: Anvisa, 2010.

12. BRASIL. Formulário Nacional. $2^{\circ}$ edição, 225p. Agência Nacional de Vigilância Sanitária. Brasília: Anvisa, 2012.

13. Kiszkiel I, Starczewska B, Leśniewska B, Późniak P. Extraction of ranitidine and nizatidine with using imidazolium ionic liquids prior spectrophotometric and chromatographic detection. J Pharm Biomed Anal. 2015; 106:85-91. DOI: 10.1016/j.jpba.2014.11.025.

14. Apostu M, Dorneanu V, Bibire N. Visible spectrophotometric assay of ranitidine. Rev Med Chir Soc Med Nat
Iasi. 2003; 107(1):214-217.

15. Sokół A, Karpińska J, Talecka R, Starczewska B. Quantification of ranitidine hydrochloride in the presence of its decomposition product by spectrophotometric methods. Application for kinetic study. Acta Pol Pharm. 2011; 68(2):169-177.

16. Basavaiah K, Somasheka BC. Argentimetric assay of ranitidine in bulk drug and in dosage forms. Ecl. Quím., São Paulo, 32(1): 19-26, 2007.

17. Espíndola VB, Cardoso TFM, Sversut RA, Carollo RH, Amaral MSD, Kassab NM. Avaliação e comparação da qualidade de medicamentos contendo cloridrato de ranitidina. Rev. Bras. Farm. 2015; 96 (2):1248-1265.

18. Santos, CSF. Medicamentos manipulados pediátricos: Desenvolvimento de formulações de ranitidina e clindamicina. [Tese]. Porto: Faculdade de Ciências da Saúde, Universidade Fernando Pessoa. 2011.

19. Ferreira AO. Guia Prático da Farmácia Magistral. 3.ed. São Paulo: Pharmabooks, 2008.

20. Tavares RC, Couto AG. Análise do perfil dos resultados de uniformidade de conteúdo de cápsulas de baixa dosagem produzidas em farmácias de manipulação de Santa Catarina. Rev. Ciênc. Farm. Básica Apl. 2011; 32 (1): 263-268.

21. Friedrich M, Primo FT, Funck JAB, Laporta LV, Alves MP, Bittencourt CF, Escarrone ALV. Avaliação da Estabilidade Físico-Química de Creme Não Iônico Inscrito no Formulário Nacional. Lat. Am. J. Pharm. 2007; 26 (4): 558-562.

22. Almeida MLC, Filho APN. Análise das cápsulas manipuladas segundo a RDC, 67/2007 da ANVISA/ MS para a garantia da qualidade. Rio de Janeiro. Rev. Bras. Farm. 2010; 91 (3):119-125.

23. BRASIL. Conselho Regional de Farmácia do Estado de São Paulo. Manual de Orientação ao Farmacêutico: Manual de Equivalência Sal/Base. 52 p. 2016.

24. Silva RL, Silva LO. Controle de qualidade quanto à determinação de peso nas cápsulas manipuladas em farmácias da cidade de Mogi Guaçu. Rev Foco. 2014; 5 (2):4160

25. Defáveri MS. Avaliação da qualidade das cápsulas de cloridrato de sibutramina manipuladas em farmácias. Disc. Scientia. Série: Ciências da Saúde. 2012; 13 (1):71-83.

26. Pinheiro GM. Determinação e avaliação de indicadores da qualidade em farmácia magistral - preparação de cápsulas gelatinosas duras. [Dissertação]. Rio de Janeiro: Faculdade de Farmácia, Universidade Federal do Rio de Janeiro. 2008.

27. Laporta LV, Brum TFD, Júnior FDRP, Santos MRD, Gonçalves CA. Validação de método analítico para avaliação da qualidade de cápsulas de cloridrato de metformina manipuladas. Rev. Ciênc. Farm. Básica Apl. 2013; 34 (2):235-244. 


\section{Inotama}

28. BRASIL. Agência Nacional de Vigilância Sanitária. Resolução RE n 899 , de 29 de maio de 2003. Determina a publicação do "Guia para validação de métodos analíticos e bioanalíticos"; fica revogada a Resolução RE $\mathrm{n}^{\circ}$ 475, de 19 de março de 2002. Diário Oficial da República Federativa do Brasil, 2 de junho de 2003.

29. Santana DPD, Guimarães SDS, Sousa GD, Melo EKSD, Albuquerque MMD, Leal LB. Avaliação dos processos de mistura de pós em farmácias magistrais utilizando planejamento fatorial: caso clonidina. Rev. Ciênc. Farm. Básica Apl. 2014; 35(1):75-79.

30. Marcatto AP, Lamim R, Block LC, Bresolin TMB. Análise de cápsulas de captopril manipuladas em farmácias. Rev. Ciênc. Farm. Básica Apl. 2005; 26(3):221-225.
31. Miotto Junior S, Adams AIH. Avaliação de cápsulas de Nifedipino manipuladas, em farmácias de Passo Fundo (RS). Rev. Infarma. 2004; 16(1-2):68-72.

32. Pissatto S, Prado JND, Morais ECD, Foppa T, Murakami FS, Silva MAS. Avaliação da Qualidade de Cápsulas de Cloridrato de Fluoxetina. Acta Farm. Bonaerense. 2006; 25(4): 550-554.

33. Meneghini LZ, Adams AIH. Avaliação físico-química de cápsulas de diazepam manipuladas em farmácias magistrais de Passo Fundo/RS. Rev. Bras. Farm. 2007; 88(2):67-70. 